Informationen der Arbeitsgemeinschaft Knochentumoren e. V.

\title{
Herbsttagung der AG Knochentumoren (ZOOM-Meeting) am 17.10.2020
}

Auch die 95. Tagung der AGKT, die für den 16. und 17. Oktober in Ulm geplant war, musste kurzfristig - ebenso wie schon die Frühjahrstagung - als Präsenzveranstaltung abgesagt werden. Die Ulmer Kollegen aus dem Institut für Pathologie um Prof. Dr. Peter Möller und Prof. Dr. Thomas Barth stellten sich jedoch umgehend auf die geänderten Bedingungen ein und ermöglichten es zusammen mit ihrem Team, die Tagung, wenn auch in etwas verkürzter Form, als virtuelle Veranstaltung nur am Samstag (17.10.) durchzuführen. Aus diesem Grund musste auch die für den 16.10. vorgesehene Diskussion von Ad-hoc-Fällen entfallen. Die wissenschaftlichen Vorträge wurden auf den Samstagvormittag verlegt und die Tagung bis 16:00 Uhr verlängert.

Die durch das Covid-19-Maßnahmen-Gesetz geänderten Regularien auch für Vereine ließen nicht nur aus Zeitgründen keine normale Mitgliederversammlung zu, sondern konnten nur als Informationsveranstaltung gelten, sodass Abstimmungen sowie Wahlen auf die nächste regulär vorbereitete Sitzung verschoben werden mussten.

In der Mitgliederinformationsveranstaltung (17.10.) wurden die für Vereine aufgrund der Pandemie geänderten Rahmenbedingungen erläutert. Diesen Umständen fielen auch die Aufnahmen neuer Mitglieder zum Opfer, da keine satzungsgemäße Abstimmung möglich war. Sie wird im Frühjahr nachgeholt werden.

Im Anschluss an die Mitgliederinformationen berichtete Prof. Dr. Sven Seiwerth über die Folgen des Erdbebens in Zagreb vom März 2020, insbesondere über die Auswirkungen auf die Zagreber Universitätsklinik und speziell auf das Institut für Pathologie, welches auf dem Campus der Universitätsklinik am schwersten betroffen war. Hier hatte die AGKT noch im Mai 2019 auf Einladung von Prof. Seiwerth ihre Frühjahrstagung abgehalten. Insgesamt wurden in Zagreb über 22000 Objekte beschädigt oder zerstört, die meisten im historischen Zentrum.

Durch tatkräftigen Einsatz und Improvisationsgeschick aller Mitarbeitenden, die zwischenzeitlich in Fluren und den wenigen nicht einsturzgefährdeten Räumen den Betrieb aufrechterhielten, und weiteren Helfern - besonders aus der Fußball-Fan-Szene - konnte das Institut für Pathologie in den folgenden 5 Monaten weitgehend wiederhergestellt werden.

An die Mitgliederversammlung schlossen sich die wissenschaftlichen Vorträge an (Zusammenfassungen sind auf den nachfolgenden Seiten zu finden):

- Primäre diffuse großzellige B-Zell-Lymphome des Knochens und chronische rekurrierende multifokale Osteomyelitis Prof. P. Möller, Institut für Pathologie der Universität Ulm

- COSS-Register und Biomaterialbank Herausforderungen und Chancen. Frau Dr. S. Hecker-Nolting, Klinikum Stuttgart-Olgaspital, und Frau Dr. S. Gottschling, Klinikum Kassel, Klinik für Onkologie und Hämatologie

- Chordom-Zellkulturmodelle: Tumorheterogenität in vitro

Dr. K. Mellert, Institut für Pathologie der Universität Ulm

Im Anschluss an die wissenschaftlichen Vorträge fand die wissenschaftliche Falldiskussion statt. Präsentiert wurden 10 Fälle. Die Diskussion weiterer 6 Fälle musste aus Zeitgründen auf den 23.1.2021 verschoben werden (Interessenten an zukünftigen Falldiskussionen können sich an das Sekretariat der AGKT - sekretariat @agkt.org - wenden).

Die Altersverteilung der besprochenen Fälle lag zwischen 13 und 80 Jahren. 6 Patienten waren jünger als 30 Jahre. 4 der 10 vorgestellten Patienten waren Frauen.
Überwiegend waren die langen Röhrenknochen betroffen ( $4 \times$ Femur, $2 \times$ Tibia), daneben die Thoraxwand, das Os ilium und die Lendenwirbelsäule. Ein Tumor (primäres BZell-Lymphom des Knochens) befiel mehrere Skelettabschnitte (Os ilium, Tibia, Femur). 4 der 10 Läsionen waren gutartig. Neben Knorpel- (4-mal) und Knochentumoren (3-mal) wurde eine aneurysmatische Knochenzyste (die von der WHO neuerdings -5 . ed./2020 - zur Gruppe der gutartigen riesenzellhaltigen Tumoren gerechnet wird), ein primäres Knochenlymphom und ein im Knochen extrem seltenes intraossäres sklerosierendes Fibrosarkom diskutiert. Besonderheiten zeigten ein Chondroblastom, welches im MRT ein exzessives Markraumödem aufwies, sowie ein hochmalignes Sarkom, das teils osteosarkomatös, teils leiomyomatös differenziert war, sodass der Tumor als primär intraossäres Knochensarkom eingeordnet wurde, seine definitive Klassifizierung aber noch offen blieb.

Den Preis für die beste Fallpräsentation erhielten die Kollegen aus München (Dr. Carolin Knebel, Dr. Ulrich Lenze, PD Dr. Carolin Mogler, PD Dr. Katja Specht und Prof. Dr. Klaus Wörtler [Orthopädische Klinik, Institut für Pathologie und Institut für Radiologie der TU München]) für die Vorstellung ihres Falles 1129 „Periostales Chondrosarkom G2“.

Die Tagung endete gegen 16:00 Uhr. Da nicht alle Fälle besprochen werden konnten, soll die Fallbesprechung am 23.1.2021 ab 9:00 Uhr wieder per ZOOMKonferenz fortgesetzt werden.

Die nächste Frühjahrstagung ist für den 16/17.4.2021 in Dresden geplant, in der Hoffnung, dass eine Präsenzveranstaltung, zumindest eine Hybridtagung möglich sein wird.

Falls Interesse besteht, als Gast an den Tagungen der AG Knochentumoren 
teilzunehmen, können weitere Informationen über das Sekretariat der AGKT in Basel (sekretariat@agkt.org) oder über die Homepage der AG Knochentumoren abgerufen werden (www.agkt.org). 